\title{
Graph of a Reflexive Game and Bélles-léttres
}

Dmitry A. Novikov

\author{
Institute of Control Sciences, Russian Academy of Sciences, \\ Moscow, Russia
}

e-mail:novikov@ipu.ru

Alexander G. Chkhartishvili

Institute of Control Sciences, Russian Academy of Sciences, Moscow, Russia

e-mail:sandro_ch@mail.ru

\begin{abstract}
:
The authors consider reflexive games that describe the interaction of subjects (agents) making decisions based on an awareness structure, i.e., a hierarchy of beliefs about essential parameters, beliefs about beliefs, and so on. It was shown that the language of graphs of reflexive games represents a convenient uniform description method for reflexion effects in bélles-léttres.
\end{abstract}

Keywords: reflexive games, awareness structure, phantom agent, graph of a reflexive game, reflexion effects in bélles-léttres.

The papers [1], [2], [3] considered a new class of game-theoretic models known as reflexive games. They describe the interaction of subjects (agents) making decisions based on an awareness structure, i.e., a hierarchy of beliefs about essential parameters, beliefs about beliefs, and so on. A solution of a reflexive game is an informational equilibrium which generalizes the definition of a Nash equilibrium from noncooperative games.

The term "reflexive games" was introduced by V. Lefebvre in 1965 (see [4]). The cited work and his other publications (e.g. [5]) represented qualitative discussions of reflexion effects in interaction among subjects (actually, no general concept of solution was suggested for this class of games). Similar remarks apply to [6], [7], [8], where a series of special cases of players' awareness was studied. The monograph [9] concentrated on systematical treatment of reflexive games and an endeavor of constructing a uniform equilibrium concept for these games.

An important concept in reflexive game analysis is the notion of a phantom agent. As a matter of fact, a phantom agent exists in the beliefs of a real or phantom agent and possesses certain awareness within the framework of these beliefs. If a reflexive game involves agent 1 and agent 2 , then agent 12 represents agent 2 in the belief of agent 1.

The mutual awareness of real and phantom agents is modeled by a directed graph (called the graph of a reflexive game). A node corresponds to a real or phantom agent; an arc between two nodes demonstrates the awareness of one agent about another agent (according to its direction). For 
instance, the graph $1 \leftrightarrow 2$ indicates that agents 1 and 2 have identical and adequate awareness about each other. Similarly, the graph $1 \leftarrow 2 \leftrightarrow 21$ means that agent 1 has adequate awareness of the opponent, whereas agent 2 gets mistaken.

Informational equilibrium evaluation requires the knowledge of the goal functions of all players. Meanwhile, the graph of a reflexive game can be constructed without specification of the goal functions of agents. Instead of the quantitative correlation of interests, this graph illustrates the qualitative correlation of awareness of reflexing agents.

Scientific literature contains a subjective description of an objective reality and strives for maximal objectivization. Contrariwise, imaginative literature (also known as bélles-léttres) naturally has reflexion - any fiction portrays a reflexive reality, i.e., results from author's reflexion. This paper provides a series of examples to elucidate the following: the graph of a reflexive game can be employed to model reflexion effects in bélles-léttres.

Example 1 ("Detective story"). Consider an investigation officer and an offender. Denote them by agents 1 and 2, respectively. Consequently, the procedure of crime detection is described by the graph of reflexive game in the form $2 \leftarrow 1 \leftrightarrow 12$ (the phantom agent 12 means that the offender strives for convincing the investigation officer in his own innocence). The fact of crime detection is described by the graph $1 \leftrightarrow 2$.

More sophisticated cases of awareness are also possible. For instance, Smerdyakov and Ivan Fedorovich (The Brothers Karamazov by F.M. Dostoevsky) possess nonidentical awareness about the murder of the father and the attitude of each other to this fact. In the eyes of Smerdyakov, the situation (the graph of reflexive game) has the form "Smerdyakov" $\leftarrow$ "Ivan Fedorovich wishes father's death" $\leftrightarrow$ "Smerdyakov is the murderer." According to Ivan Fedorovich, the situation appears as "Ivan Fedorovich" $\leftarrow$ "Smerdyakov is innocent" $\leftrightarrow$ "Ivan Fedorovich does not wish father's death."

Similar circumstances take place in Crime and Punishment. Raskol'nikov does not know that the investigation officer knows he is the murderer. Denote them by agents 1 and 2, respectively. In the mind of Raskol'nikov, the graph becomes $1 \leftarrow 12 \leftrightarrow 121$. On the other hand, the complete graph of reflexive game acquires the form presented by Fig. 1.

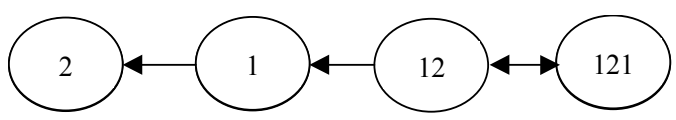

Fig. 1. The graph of reflexive game in "Detective story"

Example 2 ("Spy story-1"). Suppose that two states $(A$ and $B)$ and a public servant play the following game. The servant represents a high-level official of state $A$ and (simultaneously) an intelligencer of state $B$; this fact is unknown to state $A$. The graph of reflexive game in such situation can be found in Fig. 2. The nodes of the graph indicate the following (real and phantom) agents: 1 - state $A ; 2$ - state $B ; 3$ - the servant; 12 - state $B$ perceiving the servant as a faithful official of state $A ; 13$ - the servant as a faithful official of state $A$.

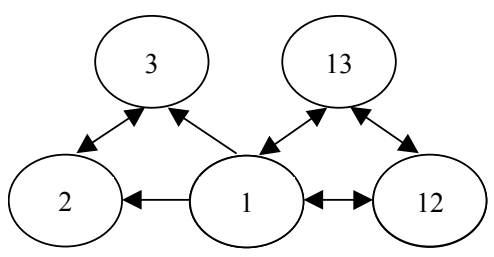

Fig. 2. The graph of reflexive game in "Spy story-1" 
Next, study a slightly complicated modification of the previous plot.

Example 3 ("Spy story-2"). The situation resembles the one described in Example 2. The difference is that the servant actually works for state $A$ (and sends specially made information to state $B$ ). In this case, the graph of reflexive game is demonstrated by Fig. 3 .

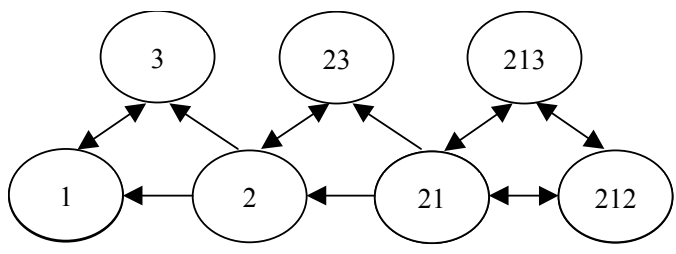

Fig. 3. The graph of reflexive game in "Spy story-2"

The nodes of this graph correspond to the following (real and phantom) agents: 1 - state $A$; 2 - state $B ; 3$ - the servant; 21 - state $A$ believing wrongly that the servant represents its official having no contacts with state $B ; 23$ - the servant working for state $B ; 212$ - state $B$ having no contacts with the servant as a high-level official of state $A ; 213$ - the servant being a faithful official of state $A$, having no contacts with state $B$.

For all examples discussed above, the maximal rank of reflexion equals 2 (and the length of the maximal sequence of indexes makes up 1). In literary works, higher reflexion ranks appear "once in a blue moon". Still, some examples do exist.

Example 4. The Emperor and the Assassin (1998), a movie directed by Kaige Chen, describes the interaction of a Chinese emperor and an assassin. The latter is sent to the former as an ambassador of a neighboring state. Meanwhile, the emperor knows that the ambassador is an assassin. And the assassin knows this, as well.

The corresponding graph of reflexive game is illustrated by Fig. 4.

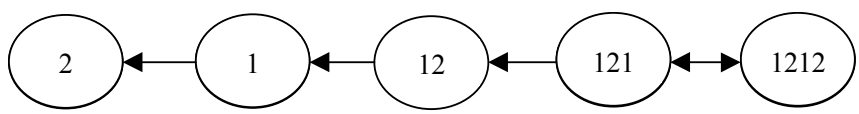

Fig. 4. The graph of reflexive game in The Emperor and the Assassin

The nodes of the graph stand for the following (real and phantom) agents: 1 - the emperor; 2 - the assassin; 12 - the assassin believing that the emperor knows nothing about him; 121 - the emperor believing that the visitor is an ambassador of a neighboring state; 1212 - an ambassador of a neighboring state.

The role of emperor's wife in the movie's intrigue can be observed in the graph of reflexive game (see agent 3 in Fig. 5). 


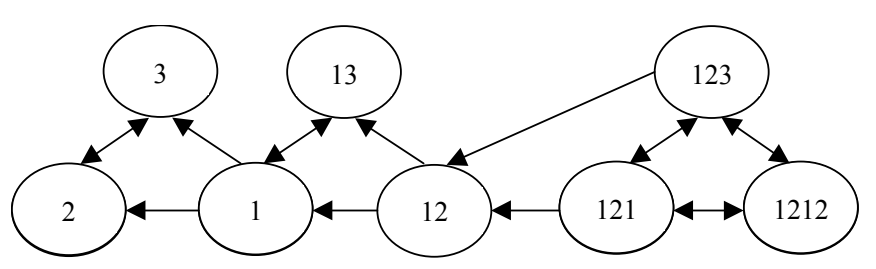

Fig. 5. The role of emperor's wife in The Emperor and the Assassin

In recent years, the existence of several reflexive (virtual, probably, embedded) realities underlies the plots of many movies. In this context, we mention The Matrix, The Thirteenth Floor, Vanilla Sky, Avalon, The Truman Show, and others. Interested readers would easily draw the corresponding graphs of reflexive games (using the approach suggested here).

The concluding example is quite a different matter.

Example 5. We cite an epigram by Coventry Patmore, known as The Kiss:

'I saw you take his kiss!' "Tis true."

'O, modesty!' 'Twas strictly kept:

He thought me asleep; at least I knew

He thought I thought he thought I slept.'

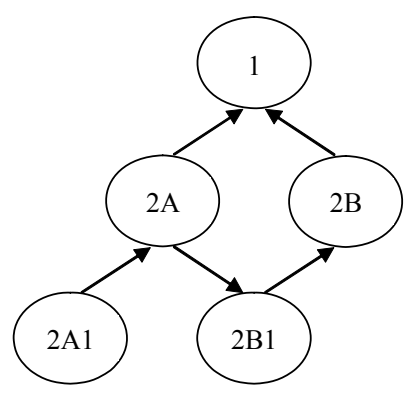

Fig. 6. The structure of lady's awareness in The Kiss

Figure 6 demonstrates the awareness structure of a lady kissed by an admirer in this epigram. Nodes of the graph correspond to the following real and phantom agents: 1 - the lady (here we have two incoming arcs, since the lady is not sure about the situation); $2 \mathrm{~A}$ - the admirer, believing that the lady is sleeping; $2 \mathrm{~B}$ - the admirer, believing that the lady thinks he considers her sleeping; 2A1 - the sleeping lady (this node admits no incoming arcs, since the lady is sleeping and performs no reflexion); $2 \mathrm{~B} 1$ - the lady, believing that the admirer thinks she is sleeping.

Therefore, the language of graphs of reflexive games represents a convenient uniform description method for reflexion effects in bélles-léttres.

\section{References:}

1. Novikov D.A. and Chkhartishvili A.G., Information Equilibrium: Punctual Structures of Information Distribution, Automation and Remote Control 2003, Vol. 64, No. 10, pp. 1609 1619. 
2. Chkhartishvili A.G. and Novikov D.A., Models of Reflexive Decision-Making, Systems Science 2004, Vol. 30, No. 2, pp. 45 - 59.

3. Chkhartishvili A.G. Reflexive Games: Transformation of Awareness Structure, Automation and Remote Control 2010, Vol. 71, No. 6, pp. 1208 - 1216.

4. Lefebvre V.A. Basic Ideas of the Reflexive Games Logic / Proc. "Problems of Systems and Structures Researches". Moscow: USSR Academy of Science, 1965 (in Russian).

5. Lefebvre V.A. Lectures on Reflexive Game Theory. - Los Angeles: Leaf \& Oaks, 2010.

6. Gorelik V.A., Kononenko A.F., Game-theoretical Models of Decision Making in Ecological and Economic Systems. Moscow: Radio and Communication, 1982 (in Russian).

7. Soros G., The Alchemy of Finance: Reading the Mind of the Market. New York: Wiley, 1994.

8. Stahl D., Wilson P. On Players Models of Other Players: Theory and Experimental Evidence, Games and Economic Behavior 1995, Vol. 10, pp. 213 - 254.

9. Novikov D.A. and Chkhartishvili A.G., Reflexion and Control. Leiden: CRC Press, 2014. 